Optinalytic (Statistical) Mirroring

\title{
Optinalytic (Statistical) Mirroring: A New Novel Approach of Measure of Dispersion
}

\author{
*Kabir Bindawa Abdullahi \\ Department of Biology, Faculty of Natural and Applied Sciences, Umaru Musa Yar'adua \\ University, P.M.B., 2218 Katsina, Katsina State, Nigeria. \\ *Correspondence: kabir.abdullahi@umyu.edu.ng ; kabirnamallam@gmail.com \\ $(+2348065995423)$
}

\begin{abstract}
The main central goal and statistical power of any statistical tool is to present the reader about the level, degree and strength of variations within or between datasets in a clear and precise interpretation that allows a researcher to make a rational and empirical conclusion. The current tools used for measure of dispersion have been challenged with some limitations. A new novel approach, called optinalytic (statistical) mirroring is proposed in this article to address some of the limitations other measure of dispersion methods have failed to solve and resolve. An optinalytic (statistical) mirrors are designed sequence images on which a sequence or set of sequences can optinalytically and intermetrically reflects to give an inferential information about their comparisons (similarity and dissimilarity). Method validation and comparisons with some most important tools of dispersion measures (e.g: variance, standard deviation, coefficient of variation, variance-to-mean ratio) was established to assess the suitability of the new proposal as an alternative measure of dispersion using different sets of logically generated univariate sequences with different problems and complications. The results of comparison shows that optinalytic (statistical) mirroring is more resistant to extreme outliers, more inferential and works efficiently with negative values with a very meaningful interpretation of result to the common understanding of a non-expert of statistics, which all other methods cannot provide.
\end{abstract}

Keywords: Statistical mirrors; central tendencies; reflector; reflecter; comparative optinalysis; inferences. 
Optinalytic (Statistical) Mirroring

\section{Introduction}

Statistics plays a vital role in all fields of research and studies ranging from culture to sciences. It helps present data precisely and draws rational, empirical and meaningful conclusions, and also makes a generalization. While presenting and analyzing data, one should be mindful of using a suitable and appropriate statistical measure (Barde and Barde, 2012). One of the essentially used descriptive statistics is the measures of central tendency (e.g mean median and mode) which are not adequate enough to describe data. One general limitation of central measure of tendency is that, two data sets can have the same mean but they can be entirely different (Manikandan, 2011). Thus to describe data in advance, one needs to understand the extent of variability which is expressed by the measures of dispersion. Range, interquartile range, and standard deviation are the three commonly used measures of dispersion (Manikandan, 2011).

The main central goal and statistical power of all statistical tools is to present the reader about the level, amount and strength of variations within or between datasets in a clear and precise interpretation that allows a researcher to make a rational and empirical conclusion. A large number of research articles (especially in biomedical studies) have statistical errors either in presentation (Cooper, et al., 2002; García-Berthou and Alcaraz, 2004) or analysis of data (Krousel-Wood et. al., 2006). Concerted efforts have been made to address these statistical errors and to improve quality of statistics (Goodman et al., 1998; Gore et al., 1992; Altman et al., 1983). Despite this, errors are still present in published articles. One such common error is use of SEM instead of SD to express variability of data (Swamy et al., 2011; Adenubi et al., 2010; Banji et al., 2011). This situational preference for the use of SD rather than SEM is usually due to presence of huge outliers in the dataset which made the data deviates a normal distribution and become meaningless when expressed in certain forms. Therefore, proper understanding and use of fundamental statistics and their application will improve reliability, interpretation, and communication of data and results to readers (Barde and Barde, 2012).

Some of the most commonly used measures of dispersion, such as variance, standard deviation, coefficient of variation, variance-to-mean ratio, standard error of mean have some disadvantages such as computational difficulty, effect and sensitivity to outliers, difficulty in results interpretation by non-expertise, and misuse of the statistical elements by researchers (Krousel-Wood et.al., 2006). Dawson and Trapp (2004) provided a clarification on when use standard deviation (when mean is used as measure of central tendency and for symmetric numerical data) and when not to be used (for ordinal data or skewed numerical data). For ordinal data or skewed numerical data, median and interquartile range should be used (Dawson and Trapp, 2004). Furthermore, measure of dispersion concentrates only at the mean but not others central measures. Up till now, to the best of my investigation, no any tool is developed to do this quantification.

Optinalysis is a new computational algorithm that intrametrically (within elements or variables) or intermetrically (between elements or variables) computes and compares two or more univariate or multiclustered or multivariate sequences as a mirror-like reflection of each other (optics-like manner), hence the name is driven. Optinalysis is based by the principles of reflection and moment about a symmetrical line which detects symmetry that reflects a similarity measurement (Abdullahi, 2019). This scientific breakthrough of optinalysis would likely to be one of the current leading concept in data science with diverse applications. 
Optinalytic (Statistical) Mirroring

Despite these limitations, standard deviation and standard error of mean remain the most used statistics to describe variations in a univariate dataset. In this article, a novel approach was developed to quantify variations in a univariate data which is less sensitive to the effect of outliers, computationally dynamic, more simple to provide interpretation and explanation, and more inferential in conclusion.

\section{Basic Concept and Assumptions about Optinalytic Mirrors}

i. In the statistical inferences of comparative optinalysis between two or more set of sequences, there can exist a reflector sequence (the object or source sequence) and reflecter sequence (the image sequence) such that the reflector sequence momentarily reflects in an optinalytic and intermetric manner on reflecter sequence for inferencial comparisons about their degree or level of similarity or dissimilarity.

ii. The reflector or source sequence is the sequence in question and reflecter sequence is the optinalytic mirror.

iii. An optinalytic mirror is therefore refers to a designed sequence image upon which a sequence or set of sequences can optinalytically and intermetrically reflects to give an inferencial information about their comparisons (similarity and dissimilarity). Comparative optinalysis that involve a reflector sequence (the object or source sequence) and reflecter sequence (the image or mirror sequence) is called optinalytic mirroring.

iv. Optinalytic mirrors in statistics are called statistical mirrors. Unlike skewness measure (which express the spread of the data around the central measure of itself), the former express and inferencially quantify the deviation (how distant) or the proximity (how close) of each sequence elements away from the central measures of tendencies. Probability level of similarity and dissimilarity expresses the proximity and deviation respectively. Moreover, it can also be used to detect a symmetric deviation or proximity of a symmetrical shapes.

v. Statistical mirror can be designed by the component of the central tendency (mean, memedian, and mode) of the distribution of sequence elements other other statistical elements of the sequence itself.

vi. In statistics, optinalytic (statistical) mirrors can be seen as meanic, medianic, modalic, maximalic, minimalic mirrors.

a. Meanic mirror: designed to expresses and inferentially quantify the deviation or proximity of each sequence elements from its mean value. It measure how far or how close each element of a dataset is from the mean value. Probability level of similarity and dissimilarity expresses the proximity and deviation respectively.

b. Medianic mirror: expresses and inferencially quantify the deviation or proximity of each sequence elements from its median value. It measure how distant or how close each element of a dataset is from the median value.

c. Modalic mirror: expresses and inferencially quantify the deviation or proximity of each sequence elements from its modal value. It measure how far or how close each element of a dataset is from the modal value.

d. Maximalic mirror: designed to expresses and inferentially quantify the deviation or proximity of each sequence elements from its maximal value. It measure how far or how close each element of a dataset is from the maximal value. 
Optinalytic (Statistical) Mirroring

e. Minimalic mirror: designed to express and inferencially quantify the deviation or proximity of each sequence elements from its minimal value. It measure how far or how close each element of a dataset is from the minimal value.

vii. Statistical mirror can therefore be structurally defined as a uniform amplification, in sequence, of a defined magnitude, called the principal element or value (e.g mean, median, and mode, minimal or maximal point of a sequence or other statistical elements) of a sequence through a defined length. The defined length of a statistical mirror must altlast be equal to the length of any object (reflector) sequence of the dataset (See Figure $1)$.

viii. In term of sequence order, statistical mirror has no define head and tail sequence, and a denotation headtail (HT) or tailhead (TH) can be used to describe the sequence.

ix. Comparative optinalysis that involve a statistical mirror sequence and its object or source sequence is called statistical mirroring.

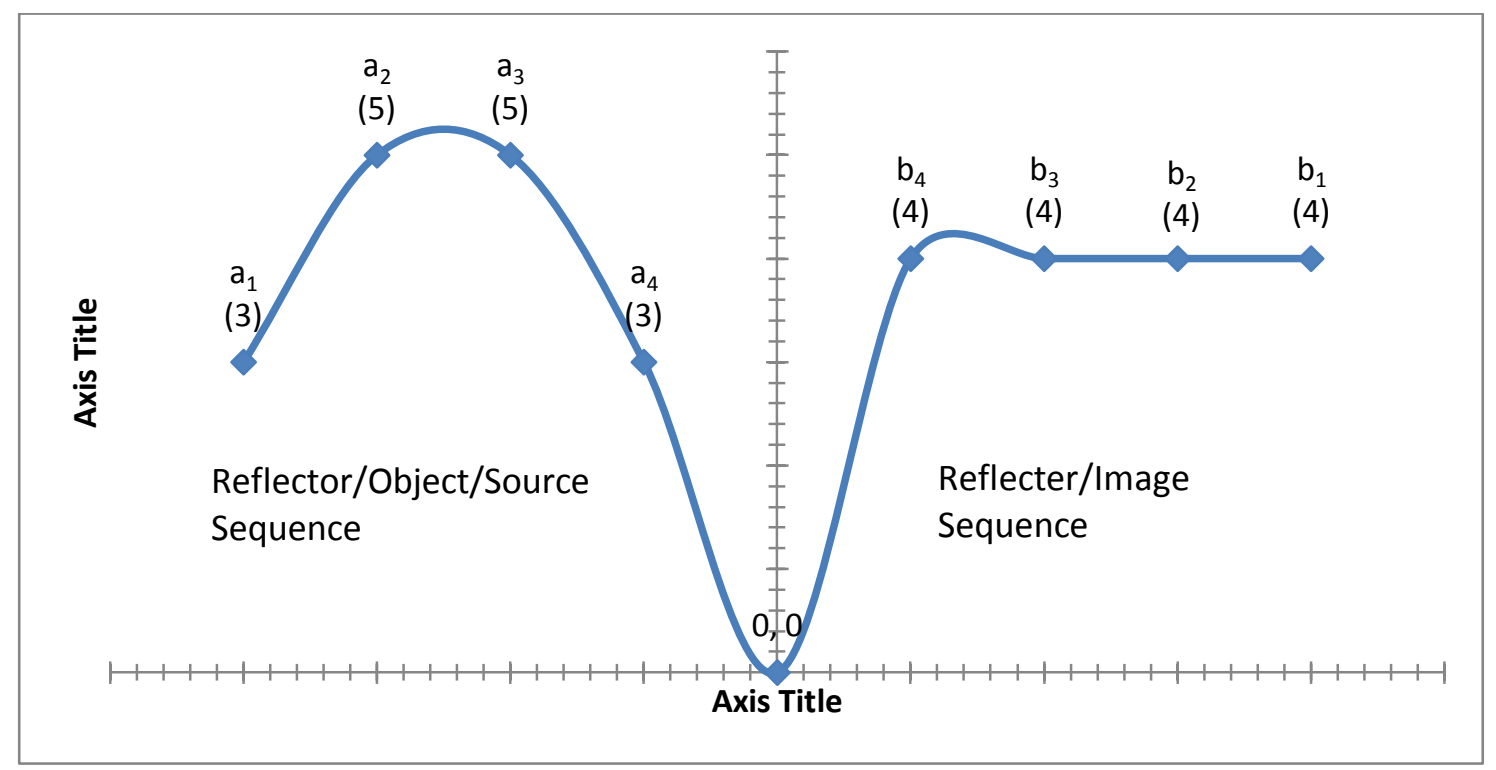

Figure 1: Pictorial model of statistical mirroring

\section{General Methodology}

In this research design, sub-methodologies were re-introduced in each specific application of statistical mirroring to provide a further details and justification about the algorithmic selection of parameters in computing the comparative optinalysis methodology. However, the general methodology presented a general approach adopted in all the specific applications.

\subsection{Datasets}

Different types of univariate and multivariate data sequence were logically generated to present different statistical variations and complications. These generated sequences were used to validate, but in application, the proposed methodology (optinalytic mirror). 
Optinalytic (Statistical) Mirroring

\subsection{Data Analysis}

Graphad Prism Statisitical Software (Version 8.2.1) was used to analyze the data for mean, median, mode, standard deviation, standard error of mean, coefficient of variation and skewness. Microsoft Excel statistical functions and cells were used to analyze the variance and variance-to-mean ratio.

Comparative optinalysis between the sequences and their designed optinalytic mirrors was performed using the method of Abdullahi (2019). Kabirian coefficient of similarity and dissimilarity, and their translated probabilities and percentages were computed. Different algorithmic parameters and argument for each application were established to suitably and appropriately analyze the data.

\subsection{Results presentation}

All results were presented in Tables.

\section{Applications and Method Validation}

\subsection{Measure of Dispersion (Scatter) from the Central Tendencies (I)}

The deviation of elements of a dataset from the central tendencies is defined by the probability or percentage of dissimilarity. And the type of statistical mirror used reflects the type of deviation been quantified. For instance:

i. Meanic mirror expresses a meanic deviation.

ii. Medianic mirror expresses a medianic deviation.

iii. Modalic mirror expresses a modalic deviation.

iv. Maximalic mirror expresses a maximalic deviation.

v. Minimalic mirror expresses a minimalic deviation.

\subsubsection{Sub-methodology I:}

Table 1 presented about 25 different sequence logically generated to express some of the different problems and complications in univariate data. For the comparative optinalysis of the datasets in Table 1, following the design of a meanic (statistical and optinalytic) mirror, the following algorithmic parameters were considered:

i. The sequence order of the random or repeated measure of the data were considered in an ascending and also in descending order. Head and tail of the sequence were denoted on the sequenced data left to the right respectively.

ii. Let each of the sequence optinalytically reflects head-to-headtail (H-HT) and also tail-toheadtail (T-HT) with each of its own meanic statistical mirror sequence, by a normalization of a zero unit, such that each sequence is intermetrically similar to its own meanic mirror sequence with a resultant Kabirain coefficient of $x$ and thus $y$ confidence of similarity. Head-to-headtail (H-HT) and tail-to-headtail (T-HT) refers to the reflection of each sequence to its meanic sequence in ascending and descending order respectively. 
Optinalytic (Statistical) Mirroring

$$
\bigwedge_{B}^{( \pm N=0)}: \int_{\in \text { (meanic mirror })}^{\in(\text { each sequence })}=x(y)
$$

The details of the data analysis and the results of comparative optinalysis were presented in Appendix A and Excel sheet 01-04 of the supplementary materials.

In this case, three (3) main attributes or criteria were used to compare the statistical power, fitness and suitability of the proposed tool (statistical mirroring) with the well-known statistical tools considered here. These criteria are (a) sensitivity and resistivity to outliers, (b) efficiency with negative values and (c) interpretation.

\subsubsection{Results and Discussion}

From Table 1, the following explanation are obtained:

Sensitivity and resistivity to outliers: All the considered and proposed statistical tools are not very efficient with large outliers except meanic deviation. Presence of large outliers make the results so large and even the calculated variance and standard deviation exceeding the mean, and therefore a valid and reliable relationship between the mean and variance or standard deviation can be rarely possible (See sequence 1F-1I, 1L-1O, 1M-1O, 1S-1W, etc). Therefore, all the considered statistical tools considered are sensitive to outliers, only meanic deviation shown a less sensitivity and much resistivity to outliers. Krousel-Wood et al. (2006) reported that variance is sensitive to outliers. If variance is sensitive to outliers, then standard deviation, standard error of mean, coefficient of variation, and variance-to-mean ratio that are derived from it are also affected.

Efficiency with negative values: All the considered and proposed statistical tools are very efficient with negative value except coefficient of variation. Presence of negative values make the calculated standard deviation exceeded the mean, and thus gives a meaningless and invalid results (See sequence 1R-1Y). In most statistical analysis, negative values are considered as outliers and seriously limit further statistical applications. Usually, normalization and transformation are otherwise normally employed to get rid of those outliers or by eliminating them from the dataset if are not many (Hancock, et al., 1988).

Interpretation: Confidence level is the easiest to give an inferential interpretation and draw a conclusion. Although coefficient of variation, and variance-to-mean ratio are assumed to provide a clear inferential (probabilistic) conclusion, but all have failed in some cases with much ambiguity. Standard error of mean can be used to further construct a confidence interval, but at a ground stage of its results, it makes no inferential meaning. All the considered and proposed statistical tools provided a clear magnitude and information about the variation and dispersion of the datasets, but a very clear problems to all the tools except meanic deviation is valid and reliable (without ambiguity) interpretation of the result to the common understanding of a nonexpert of statistics (See sequence 1F-1I, 1L-1O, 1N-1O, and 1R-1Y, etc). Again, when we look at sequence $1 \mathrm{~N}$, we can question how valid and reliable is the result. It is practically impossible to obtain a $100 \%$ deviation just by the presence of positive outliers, but we can logically accept that a $100 \%$ deviation can be possible by a negative outliers equal in magnitude to its positive deviants (See sequence 1R with 100\% deviation). 
Optinalytic (Statistical) Mirroring

Other observations: The method of meanic deviation in relation to the sequence orders (ascending and descending order), in some cases, may give the same comparative results, but in other cases, different results were obtained especially when the sequence elements includes a large negative outliers. However, looking at the skewness level, we cannot make a conclusion based on this problems that discrepancies related to the other methods were due to skewness. From the results in Table 1, only sequences 1D-1G have a skewness less than 10 digits roundedup value. Coefficient of variation is shown in Table 1 to be more stable with less ambiguity than variance-to-mean ratio, which is likely due to difference in the sensitivity level to outliers by the variance and standard deviation that derived them respectively (Krousel-Wood et al., 2006). 


\section{Optinalytic (Statistical) Mirroring}

Table 1: Comparative results between some well-known statistical tools and meanic deviation

\begin{tabular}{|c|c|c|c|c|c|c|c|c|c|c|}
\hline Seq. & Ascending sequence order & Mean & Var. & SD & SEM & CV (\%) & VMR & Skewness & MD (\%) & MD (\%) \\
\hline & & & & & & & & (Pearson) & H-HT & T-HT \\
\hline $\mathrm{TA}$ & ${ }^{(\mathrm{H})}(1,2,3,4,5,6,7,8,9,10,11,12)^{(\mathrm{T})}$ & 6.5 & 13.00 & 3.606 & 1.041 & 55.47 & $2.00 *$ & 0 & 24.72 & 24.72 \\
\hline $1 \mathrm{~B}$ & (H) $(3,3,3,3,3,3,3,3,3,3,3,3)^{(\mathrm{T})}$ & 3 & 0.00 & 0 & 0 & 0.000 & 0.00 & NES & 0.00 & 0.00 \\
\hline $1 \mathrm{C}$ & (H) $(1,2,3,4,4,4,4,4,4,5,6,7)^{(\mathrm{T})}$ & 4 & 2.55 & 1.595 & 0.4606 & 39.89 & 0.638 & 0 & 17.01 & 17.01 \\
\hline $1 \mathrm{D}$ & (H) $(3,4,5,8,8,8,8,8,8,8,8,8)^{(\mathrm{T})}$ & 7 & 3.45 & 1.859 & 0.5365 & 26.55 & 0.493 & -1.529 & 9.76 & 9.76 \\
\hline $1 \mathrm{E}$ & (H) $(8,8,8,8,8,8,8,8,8,11,12,13)^{(\mathrm{T})}$ & 9 & 3.45 & 1.859 & 0.5365 & 20.65 & 0.383 & 1.529 & 7.67 & 7.67 \\
\hline $1 \mathrm{~F}$ & (H) $(2,7,7,7,7,7,7,7,7,7,7,35)^{(\mathrm{T})}$ & 8.917 & 69.54 & 8.339 & 2.407 & 93.52 & $7.799 *$ & 3.263 & 23.08 & 23.08 \\
\hline $1 \mathrm{G}$ & (H) $(7,7,7,7,7,7,7,7,7,7,7,35)^{(\mathrm{T})}$ & 9.333 & 65.33 & 8.083 & 2.333 & 86.60 & $7.000 *$ & 3.464 & 19.13 & 19.13 \\
\hline $1 \mathrm{H}$ & (H) $(2,2,2,2,2,2,9,9,9,9,9,9)^{(\mathrm{T})}$ & 5.5 & 13.36 & 3.656 & 1.055 & 66.47 & $2.429 *$ & 0 & 25.61 & 25.61 \\
\hline 1I & ${ }^{(\mathrm{H})}(2,2,2,2,2,2,7,7,7,7,7,7)^{(\mathrm{T})}$ & 4.5 & 6.82 & 2.611 & 0.7538 & 58.03 & $1.516^{*}$ & 0 & 22.73 & 22.73 \\
\hline $1 \mathrm{~J}$ & (H) $(4,4,4,4,4,4,4,4,4,4)^{(\mathrm{T})}$ & 4 & 0.00 & 0 & 0 & 0.000 & 0.000 & NES & 0.00 & 0.00 \\
\hline $1 \mathrm{~K}$ & (H) $(4,4,4,4,4,8,8,8,8,8)^{(\mathrm{T})}$ & 6 & 4.44 & 2.108 & 0.6667 & 35.14 & 0.740 & 0 & 14.08 & 14.08 \\
\hline $1 \mathrm{~L}$ & (H) $(4,4,4,4,4,16,16,16,16,16)^{(\mathrm{T})}$ & 10 & 40.00 & 6.325 & 2 & 63.25 & $4.000^{*}$ & 0 & 24.00 & 24.00 \\
\hline $1 \mathrm{M}$ & (H) $(4,4,4,4,4,64,64,64,64,64)^{(\mathrm{T})}$ & 34 & 1000.00 & 31.62 & 10 & 93.01 & $29.412 *$ & 0 & 33.41 & 33.41 \\
\hline $1 \mathrm{~N}$ & (H) $(4,4,4,4,4,152,152,152,152,152)^{(\mathrm{T})}$ & 78 & 6084.44 & 78 & 24.67 & $100.0 *$ & $78.006^{*}$ & 0 & 35.47 & 35.47 \\
\hline 10 & (H) $(4,4,4,4,4,256,256,256,256,256)^{(\mathrm{T})}$ & 130 & 17640.00 & 132.8 & 42 & $102.2 *$ & $135.692 *$ & 0 & 36.10 & 36.10 \\
\hline $1 \mathrm{P}$ & (H) $(4,4,4,4,4,3.999,3.999,3.999,3.999,3.999)^{(\mathrm{T})}$ & 4 & 0.00 & 0.000527 & 0.000167 & 0.01318 & 0.000 & $-1.6 \mathrm{E}-12$ & 0.01 & 0.01 \\
\hline $1 \mathrm{Q}$ & (H) $(4,4,4,4,4,4.001,4.001,4.001,4.001,4.001)^{(\mathrm{T})}$ & 4.001 & 0.00 & 0.000527 & 0.000167 & 0.01317 & 0.000 & $-3.2 \mathrm{E}-12$ & 0.01 & 0.01 \\
\hline $1 \mathrm{R}$ & (H) $(4,4,4,4,4,-4,-4,-4,-4,-4)^{(\mathrm{T})}$ & 0 & 17.78 & 4.216 & 1.333 & NES & NES & 0 & 100.00 & 100.00 \\
\hline is & (H) $(-8,-8,-8,-8,-8,4,4,4,4,4)^{(\mathrm{T})}$ & -2 & 40.00 & 6.325 & 2 & $316.2 *$ & $-20.000 *$ & 0 & 81.08 & 81.08 \\
\hline $1 \mathrm{~T}$ & (H) $(-16,-16,-16,-16,-16,4,4,4,4,4)^{(\mathrm{T})}$ & -6 & 111.11 & 10.54 & 3.333 & $175.7^{*}$ & $-18.518 *$ & 0 & 54.95 & 54.95 \\
\hline $1 \mathrm{U}$ & (H) $(-64,-64,-64,-64,-64,4,4,4,4,4)^{(\mathrm{T})}$ & -30 & 1284.44 & 35.84 & 11.33 & $119.5^{*}$ & $-42.815^{*}$ & 0 & 40.96 & 40.96 \\
\hline $1 \mathrm{~V}$ & (H) $(-152,-152,-152,-152,-152,4,4,4,4,4)^{(\mathrm{T})}$ & -74 & 6760.00 & 82.22 & 26 & $111.1^{*}$ & $-91.351 *$ & 0 & 45.99 & 37.05 \\
\hline $1 \mathrm{~W}$ & (H) $(-256,-256,-256,-256,-256,4,4,4,4,4)^{(\mathrm{T})}$ & -126 & 18777.78 & 137 & 43.33 & $108.8^{*}$ & $-149.030 *$ & 0 & 47.52 & 35.77 \\
\hline $1 \mathrm{X}$ & (H) $(-3.999,-3.999,-3.999,-3.999,-3.999,4,4,4,4,4)^{(\mathrm{T})}$ & 0.0005 & 17.77 & 4.216 & 1.333 & $843169^{*}$ & $35540.00 *$ & $2.11 \mathrm{E}-16$ & 27.83 & 72.26 \\
\hline $1 \mathrm{Y}$ & (H) $(-4.001,-4.001,-4.001,-4.001,-4.001,4,4,4,4,4)^{(\mathrm{T})}$ & -0.0005 & 17.78 & 4.217 & 1.334 & $843379 *$ & $-35560.00^{*}$ & $4.21 \mathrm{E}-16$ & 27.82 & 72.26 \\
\hline
\end{tabular}

*= Ambiguous result; Var $=$ Variance $\mathrm{SD}=$ Standard deviation; $\mathrm{SEM}=$ Standard error of mean $\mathrm{CV}=\mathrm{Coefficient}$ of variation;

$\mathrm{VRM}=$ Variance-to-mean ratio; $\mathrm{K}_{\mathrm{c}}=$ Kabirian coefficient of similarity; $\mathrm{MD}=$ Meanic deviation; NES = Not efficient to solve 
Optinalytic (Statistical) Mirroring

\subsection{Measure of Dispersion (Scatter) from the Central Tendencies (II)}

\subsubsection{Sub-methodology II:}

Table 2 presented a generated frequency distribution of individuals in different age groups from four (4) different populations A-D. For the comparative optinalysis of the datasets in Table 2, following the design of a meanic, medianic, modalic, maximalic and minimalic mirrors, the following algorithmic parameters were considered:

i. The ascending sequence order was used to sequence the individuals' frequencies, and the inherent order by the age groups is disregarded. The denotation of the head to tail is by sequenced order from smallest to largest respectively.

ii. Let each of the sequence of the populations A-D optinalytically reflects head-to-headtail (H-HT) and also tail-to-headtail (T-HT) with each of its own meanic, medianic, modalic, maximalic and minimalic statistical mirror sequences, by a normalization of a zero unit, such that sequence of response scores of each of the populations A-D is intermetrically similar to each of their own respective statistical mirrors sequence with a resultant Kabirain coefficient of $x$ and thus $y$ confidence of similarity.

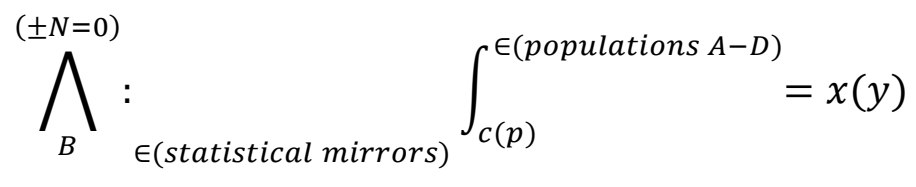

The details of the data analysis and the results of comparative optinalysis were presented in Appendix B and Excel sheet 05 of the supplementary materials.

In this case, three (3) main attributes or criteria were used to compare the statistical power, fitness and suitability of the proposed tool (statistical mirroring) with the well-known statistical tools considered here. These criteria are (a) trend of variation, (b) sensitivity and resistivity to outliers, (c) interpretation.

\subsubsection{Results and Discussion}

From Table 2, the following explanation are obtained:

\subsubsection{Deviations from the Mean}

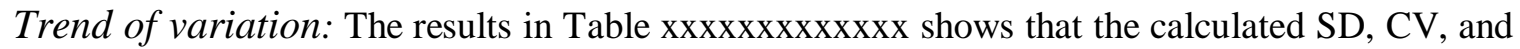
meanic deviations follows the same decreasing order between the populations as $B \rightarrow C \rightarrow A \rightarrow D$. This shows that all the methods give same trend of variation.

Sensitivity and resistivity to outliers: Although the direction is predicted, but coefficient of variation at population B looks ambiguous and meaningless unlike the meanic deviation. The presence of outliers is a possible effect that make the calculated standard deviation more than the mean. Therefore, meanic deviation is more resistive to outliers than coefficient of variation and

Interpretation: As previously explained, the considered and proposed statistical tools provided a clear magnitude and information about the variation and dispersion of the datasets, but a very clear problems to all the tools except coefficient of variation and meanic deviation is interpretation of the result to the common understanding of a non-expert of statistics. 
Optinalytic (Statistical) Mirroring

Other Observations: Looking at these three approaches (SD, CV, and meanic deviation), it can be explained that meanic deviation is another suitable alternative which is more better and precise over the other well-known measures of dispersion from the mean, because all the results are reliable, more inferential and can be easily understood by a non-expert of statistics.

\subsubsection{Deviations from the Median, Mode, Maximum and Minimum}

A clear statistical tools is not known to measure the dispersion of elements from the median, mode, maximum and minimum. Nevertheless, range is obtain used to show the limit at the boundaries of the dataset, it is not showing the strength of their deviations. By comparing the ranges and the alikeness of the intermediate elements in each populations, it can be explained that both the maximalic and minimalic deviations give a quantitative justification of the extent of variations.

Table 2: Statistical mirroring and some measure of dispersion

\begin{tabular}{|c|c|c|c|c|}
\hline \multirow[b]{2}{*}{ Age groups } & \multicolumn{4}{|c|}{ Frequency of Individuals Age Group } \\
\hline & Population A & Population B & Population C & Population D \\
\hline (H) $1-5$ & 100 & 10 & 100 & 100 \\
\hline $6-10$ & 373 & 77 & 696 & 396 \\
\hline $11-15$ & 447 & 44 & 737 & 737 \\
\hline $16-20$ & 782 & 78 & 537 & 537 \\
\hline $21-25$ & 210 & 281 & 786 & 586 \\
\hline $26-30$ & 638 & 981 & 281 & 281 \\
\hline $31-35$ & 537 & 537 & 78 & 278 \\
\hline $36-40$ & 223 & 637 & 544 & 544 \\
\hline $41-45$ & 186 & 986 & 237 & 237 \\
\hline $46-50^{(\mathrm{T})}$ & 100 & 100 & 10 & 310 \\
\hline Parameters & \multicolumn{4}{|c|}{$\begin{array}{c}\text { Results } \\
\end{array}$} \\
\hline Standard deviation $(\mathrm{M} \pm \mathrm{SD})$ & $359.6 \pm 236$ & $373.1 \pm 385.8$ & $400.6 \pm 293.7$ & $400.6 \pm 194.8$ \\
\hline Coefficient of variation $(\mathrm{M} \pm \mathrm{CV})$ & $359.6 \pm 65.62 \%$ & $373.1 \pm 103.4 \% *$ & $400.6 \pm 73.31 \%$ & $400.6 \pm 48.62 \%$ \\
\hline Meanic deviation $\left(\mathrm{M} \pm \mathrm{P}_{\text {Dsim }}\right)$ & $359.6 \pm 27.33 \%$ & $373.1 \pm 38.71 \%$ & $400.6 \pm 30.33 \%$ & $400.6 \pm 21.04 \%$ \\
\hline Medianic deviation $\left(\mathrm{Me} \pm \mathrm{P}_{\text {Dsim }}\right)$ & $298 \pm 14.71 \%$ & $190.5 \pm 98.77 \%$ & $409 \pm 31.55 \%$ & $353 \pm 11.64 \%$ \\
\hline Modalic deviation $\left(\mathrm{Mo} \pm \mathrm{P}_{\text {Dsim }}\right)$ & $782 \pm 63.92 \%$ & $986 \pm 73.64 \%$ & $786 \pm 61.64 \%$ & $737 \pm 54.91 \%$ \\
\hline Maximalic deviation $\left(\mathrm{Ma} \pm \mathrm{P}_{\text {Dsim }}\right)$ & $782 \pm 63.92 \%$ & $986 \pm 73.64 \%$ & $786 \pm 61.64 \%$ & $737 \pm 54.91 \%$ \\
\hline Minimalic deviation $\left(\mathrm{Mi} \pm \mathrm{P}_{\text {Dsim }}\right)$ & $100 \pm 48.16 \%$ & $10 \pm 64.89 \%$ & $10 \pm 75.19 \%$ & $100 \pm 58.39 \%$ \\
\hline
\end{tabular}

*= Ambiguous result; $\mathrm{M}=$ Mean; Me = Median; Mo = Mode; Ma =Maximum; Mi =Minimum.

\subsection{Symmetric Deviations of a Symmetric Shape}

Symmetry lies everywhere, but how much do we know about the uniform deviation of all the sequence elements by a certain factor away from a known or reference shape sequence through magnification, either positive or negative. One way of doing this is to compare the magnified shape sequence with the original shape sequence. Another interesting fact about a statistical mirror is that, the whole sequence elements of a given shape can be represented by its meanic mirror (See Figure 3).

\subsubsection{Sub-methodology III:}

To prove this representation, we detected the deviations of a symmetrical shape sequence $A$ by the effect of a magnification factor $x$ on the symmetrical shape sequence elements, $x[\in(A)]$. 
Optinalytic (Statistical) Mirroring

Let $A$ be the resolved sequence of a symmetric shape of an object, let $B$ be the statistical meanic mirror of sequence $A$ and let $x(A)$ be the magnified shape sequence $A$.

$\mathrm{A}=(8,4,1,4,8)$

$\mathrm{B}=(5,5,5,5,5)$

The details of the data analysis and the results of comparative optinalysis were presented in Appendix C and Excel sheet 06-07 of the supplementary materials.

In this case, two (2) main attributes or criteria were used to prove the statistical power, fitness and suitability of the proposed tool. These criteria are (a) magnification effect (b) Results consistency in a reverse effect of magnification (c) representation potential of statistical mirrors.

\subsubsection{Results and Discussion}

From Table 3, the following explanation are obtained:

Magnification effect: The magnification factors exactly correspond to the outcomes comparative optinalysis. This proves the validity and reliability results and methodology.

Results consistency in a reverse effect of magnification: The results of positive and negative magnification by same factor give the same results. This proves the validity and reliability results and methodology.

Representation potential of statistical mirrors: Results consistency between comparative optinalysis and statistical mirroring shows the potential power of sequence representation by statistical mirror. 
Optinalytic (Statistical) Mirroring

Table 3: Measure of factorial deviation of a symmetrical shape by statistical mirroring

\begin{tabular}{|c|c|c|c|c|c|}
\hline & \multicolumn{5}{|c|}{ Comparative Optinalysis } \\
\hline & $x(A):(A)$ & $x(A):(B)$ & & $x(A):(A)$ & $x(A):(B)$ \\
\hline $\begin{array}{l}\text { Magnification } \\
\text { Factor }(\boldsymbol{x})\end{array}$ & $\bigwedge_{B}^{( \pm N v=0)}: \int_{\in(A)}^{x[E(A)]}=K_{c}(? \%$ Sim. $)$ & $\bigwedge_{B}^{( \pm N v=0)}: \int_{\in(B)}^{x[E(A)]}=K_{c}(? \%$ Sim. $)$ & $\begin{array}{l}\text { Magnification } \\
\text { Factor }(x)\end{array}$ & $\bigwedge_{B}^{( \pm N v=0)}: \int_{\in(A)}^{x[\in(A)]}=K_{c}(? \% \operatorname{Sim})$. & $\bigwedge_{B}^{( \pm N v=0)}: \int_{x[\in(B)]}^{\in(A)}=K_{c(p)}(? \% \operatorname{Sim})$. \\
\hline$\times 2$ & $1.200000(50.00)$ & $1.200000(50.00)$ & $\times 1 / 2$ & $0.857143(50.00)$ & $0.857143(50.00)$ \\
\hline$\times 3$ & $1.333333(33.33)$ & $1.333333(33.33)$ & $\times 1 / 3$ & $0.800000(33.33)$ & $0.800000(33.33)$ \\
\hline$\times 4$ & $1.428571(25.00)$ & $1.428571(25.00)$ & $\times 1 / 4$ & $0.769231(25.00)$ & $0.769231(25.00)$ \\
\hline$\times 5$ & $1.500000(20.00)$ & $1.500000(20.00)$ & $\times 1 / 5$ & $0.750000(20.00)$ & $0.750000(20.00)$ \\
\hline$\times 6$ & $1.555556(16.67)$ & $1.555556(16.67)$ & $\times 1 / 6$ & $0.736842(16.67)$ & $0.736842(16.67)$ \\
\hline$\times 7$ & $1.600000(14.29)$ & $1.600000(14.29)$ & $\times 1 / 7$ & $0.727273(14.29)$ & $0.727273(14.29)$ \\
\hline$\times 8$ & $1.636364(12.50)$ & $1.636364(12.50)$ & $\times 1 / 8$ & $0.720000(12.50)$ & $0.720000(12.50)$ \\
\hline$\times 9$ & $1.666667(11.11)$ & $1.666667(11.11)$ & $\times 1 / 9$ & $0.714286(11.11)$ & $0.714286(11.11)$ \\
\hline$\times 10$ & $1.692308(10.00)$ & $1.692308(10.00)$ & $\times 1 / 10$ & $0.709677(10.00)$ & $0.709677(10.00)$ \\
\hline
\end{tabular}


Optinalytic (Statistical) Mirroring

\section{Conclusion}

Considering all the applications and results of comparisons here studied, it is concluded that optinalytic (statistical) mirroring is more resistant to extreme outliers, more inferential and works efficiently with negative values with a very meaningful interpretation of result to the common understanding of a non-expert of statistics, which all other methods cannot provide. It is therefore a very suitable approach of measure of dispersion of univariate datasets.

\section{Conflict of interest}

The author declares no conflict of interest.

\section{References}

Abdullahi, K. B. (2019). Optinalysis: A New Approach of Symmetry Detection and Similarity Measurement through a Looking-Glass. Preprints, 2019050295. doi: 10.20944/preprints201905.0295.v2

Adenubi, O. T., Raji, Y., Awe, E. O., \& Makinde, J. M. (2010). The effect of the aqueous extract of the leaves of boerhavia diffusa linn. on semen and testicular morphology of male Wistar rats. Science World Journal, 5(2).

Altman, D. G., Gore, S. M., Gardner, M. J., \& Pocock, S. J. (1992). Statistical guidelines for contributors to medical journals.

Banji, D., Pinnapureddy, J., Banji, O. J., Kumar, A. R., \& Reddy, K. N. (2011). Evaluation of the concomitant use of methotrexate and curcumin on Freund's complete adjuvant-induced arthritis and hematological indices in rats. Indian journal of pharmacology, 43(5), 546.

Barde, M. P., \& Barde, P. J. (2012). What to use to express the variability of data: Standard deviation or standard error of mean?. Perspectives in clinical research, 3(3), 113.

Bihaqi, S. W., Singh, A. P., \& Tiwari, M. (2011). In vivo investigation of the neuroprotective property of Convolvulus pluricaulis in scopolamine-induced cognitive impairments in Wistar rats. Indian journal of pharmacology, 43(5), 520.

Cooper, R. J., Schriger, D. L., \& Close, R. J. (2002). Graphical literacy: the quality of graphs in a large-circulation journal. Annals of emergency medicine, 40(3), 317-322.

Dawson, B., Trapp, R.G. (2004). Basic and Clinical Biostatistics. 4th ed. New York: Mc-Graw Hill.

García-Berthou, E., \& Alcaraz, C. (2004). Incongruence between test statistics and P values in medical papers. BMC medical research methodology, 4(1), 13.

Goodman, S. N., Altman, D. G., \& George, S. L. (1998). Statistical reviewing policies of medical journals. Journal of general internal medicine, 13(11), 753-756.

Gore, S. M., Jones, G., \& Thompson, S. G. (1992). The Lancet's statistical review process: areas for improvement by authors. The Lancet, 340(8811), 100-102. 
Optinalytic (Statistical) Mirroring

Hancock, A. A., Bush, E. N., Stanisic, D., Kyncl, J. J., \& Lin, C. T. (1988). Data normalization before statistical analysis: keeping the horse before the cart. Trends in pharmacological sciences, 9(1), 29-32.

Krousel-Wood, M. A., Chambers, R. B., \& Muntner, P. (2006). Clinicians' Guide to Statistics for Medical Practice and Research: Part I. The Ochsner Journal, 6(2), 68.

Manikandan, S. (2011). Measures of dispersion. Journal of Pharmacology and Pharmacotherapeutics, 2(4), 315.

Swamy, A. V., Wangikar, U., Koti, B. C., Thippeswamy, A. H. M., Ronad, P. M., \& Manjula, D. V. (2011). Cardioprotective effect of ascorbic acid on doxorubicin-induced myocardial toxicity in rats. Indian journal of pharmacology, 43(5), 507. 
Optinalytic (Statistical) Mirroring

\section{Appendix A}

Table A1: Sequences and their Designed Statistical Meanic Mirrors

\begin{tabular}{|c|c|c|c|}
\hline & & & Optinalysis (Statistical) Mirror \\
\hline Seq. & Ascending sequence order & Mean & Meanic Mirror \\
\hline TA & $(\mathrm{H})(1,2,3,4,5,6,7,8,9,10,11,12)^{(\mathrm{T})}$ & 6.5 & $(6.5,6.5,6.5,6.5,6.5,6.5,6.5,6.5,6.5,6.5,6.5,6.5)$ \\
\hline 1B & (H) $(3,3,3,3,3,3,3,3,3,3,3,3)^{(\mathrm{T})}$ & 3 & $(3,3,3,3,3,3,3,3,3,3,3,3)$ \\
\hline $1 \mathrm{C}$ & ${ }^{(\mathrm{H})}(1,2,3,4,4,4,4,4,4,5,6,7)^{(\mathrm{T})}$ & 4 & $(4,4,4,4,4,4,4,4,4,4,4,4)$ \\
\hline 1D & (H) $(3,4,5,8,8,8,8,8,8,8,8,8)^{(\mathrm{T})}$ & 7 & $(7,7,7,7,7,7,7,7,7,7,7,7)$ \\
\hline $1 \mathrm{E}$ & (H) $(8,8,8,8,8,8,8,8,8,11,12,13)^{(\mathrm{T})}$ & 9 & $(9,9,9,9,9,9,9,9,9,9,9,9)$ \\
\hline $1 \mathrm{~F}$ & $(\mathrm{H})(2,7,7,7,7,7,7,7,7,7,7,35)^{(\mathrm{T})}$ & 8.917 & $(8.917,8.917,8.917,8.917,8.917,8.917,8.917,8.917,8.917,8.917,8.917,8.917)$ \\
\hline $1 \mathrm{G}$ & (H) $(7,7,7,7,7,7,7,7,7,7,7,35)^{(\mathrm{T})}$ & 9.333 & $(9.333,9.333,9.333,9.333,9.333,9.333,9.333,9.333,9.333,9.333,9.333,9.333)$ \\
\hline $1 \mathrm{H}$ & (H) $(2,2,2,2,2,2,9,9,9,9,9,9)^{(\mathrm{T})}$ & 5.5 & $(5.5,5.5,5.5,5.5,5.5,5.5,5.5,5.5,5.5,5.5,5.5,5.5)$ \\
\hline 1I & ${ }^{(\mathrm{H})}(2,2,2,2,2,2,7,7,7,7,7,7)^{(\mathrm{T})}$ & 4.5 & $(4.5,4.5,4.5,4.5,4.5,4.5,4.5,4.5,4.5,4.5,4.5,4.5)$ \\
\hline $1 \mathrm{~J}$ & $(4,4,4,4,4,4,4,4,4,4)^{(\mathrm{T})}$ & 4 & $(4,4,4,4,4,4,4,4,4,4)$ \\
\hline $1 \mathrm{~K}$ & (H) $(4,4,4,4,4,8,8,8,8,8)^{(\mathrm{T})}$ & 6 & $(6,6,6,6,6,6,6,6,6,6)$ \\
\hline $1 \mathrm{~L}$ & (H) $(4,4,4,4,4,16,16,16,16,16)^{(\mathrm{T})}$ & 10 & $(10,10,10,10,10,10,10,10,10,10)$ \\
\hline $1 \mathrm{M}$ & (H) $(4,4,4,4,4,64,64,64,64,64)^{(\mathrm{T})}$ & 34 & $(34,34,34,34,34,34,34,34,34,34)$ \\
\hline $1 \mathrm{~N}$ & (H) $(4,4,4,4,4,152,152,152,152,152)^{(\mathrm{T})}$ & 78 & $(78,78,78,78,78,78,78,78,78,78)$ \\
\hline 10 & (H) $(4,4,4,4,4,256,256,256,256,256)^{(\mathrm{T})}$ & 130 & $(130,130,130,130,130,130,130,130,130,130)$ \\
\hline $1 \mathrm{P}$ & (H) $(4,4,4,4,4,3.999,3.999,3.999,3.999,3.999)^{(\mathrm{T})}$ & 4 & $(4,4,4,4,4,4,4,4,4,4)$ \\
\hline 1Q & (H) $(4,4,4,4,4,4.001,4.001,4.001,4.001,4.001)^{(\mathrm{T})}$ & 4.001 & $(4.001,4.001,4.001,4.001,4.001,4.001,4.001,4.001,4.001,4.001)$ \\
\hline $1 \mathrm{R}$ & $(-4,-4,-4,-4,-4,4,4,4,4,4)^{(\mathrm{T})}$ & 0 & $(0,0,0,0,0,0,0,0,0,0)$ \\
\hline $1 \mathrm{~S}$ & (H) $(-8,-8,-8,-8,-8,4,4,4,4,4)^{(\mathrm{T})}$ & -2 & $(-2,-2,-2,-2,-2,-2,-2,-2,-2,-2)$ \\
\hline $1 \mathrm{~T}$ & ${ }^{(\mathrm{H})}(-16,-16,-16,-16,-16,4,4,4,4,4)^{(\mathrm{T})}$ & -6 & $(-6,-6,-6,-6,-6,-6,-6,-6,-6,-6)$ \\
\hline $1 \mathrm{U}$ & (H) $(-64,-64,-64,-64,-64,4,4,4,4,4)^{(\mathrm{T})}$ & -30 & $(-30,-30,-30,-30,-30,-30,-30,-30,-30,-30)$ \\
\hline $1 \mathrm{~V}$ & (H) $(-152,-152,-152,-152,-152,4,4,4,4,4)^{(\mathrm{T})}$ & -74 & $(-74,-74,-74,-74,-74,-74,-74,-74,-74,-74)$ \\
\hline $1 \mathrm{~W}$ & $(\mathrm{H})(-256,-256,-256,-256,-256,4,4,4,4,4)^{(\mathrm{T})}$ & -126 & $(-126,-126,-126,-126,-126,-126,-126,-126,-126,-126)$ \\
\hline $1 \mathrm{X}$ & (H) $(-3.999,-3.999,-3.999,-3.999,-3.999,4,4,4,4,4)^{(\mathrm{T})}$ & 0.0005 & $(0.0005,0.0005,0.0005,0.0005,0.0005,0.0005,0.0005,0.0005,0.0005,0.0005)$ \\
\hline $1 \mathrm{Y}$ & (H) $(-4.001,-4.001,-4.001,-4.001,-4.001,4,4,4,4,4)^{(\mathrm{T})}$ & -0.0005 & $(-0.0005,-0.0005,-0.0005,-0.0005,-0.0005,-0.0005,-0.0005,-0.0005,-0.0005,-0.0005)$ \\
\hline
\end{tabular}


Optinalytic (Statistical) Mirroring

Table A2: Results of Statistical Mirroring

\begin{tabular}{|c|c|c|c|c|c|c|c|}
\hline \multirow{3}{*}{ Seq. } & \multirow{3}{*}{ Ascending sequence order } & \multicolumn{6}{|c|}{ Comparative Optinalysis } \\
\hline & & \multicolumn{3}{|c|}{ Head-to-headtail Pairing } & \multicolumn{3}{|c|}{ Tail-to-headtail Pairing } \\
\hline & & $\mathbf{K}_{\mathbf{C}}$ & \%Sim. & \%Dsim. & $\mathbf{K}_{\mathbf{C}}$ & \%Sim. & \%Dsim. \\
\hline $2 \mathrm{~A}$ & ${ }^{(\mathrm{H})} 1,2,3,4,5,6,7,8,9,10,11,12^{(\mathrm{T})}$ & 0.934132 & 75.28 & 24.72 & 1.075862 & 75.28 & 24.72 \\
\hline $2 \mathrm{~B}$ & (H) $3,3,3,3,3,3,3,3,3,3,3,3^{(\mathrm{T})}$ & 1.000000 & 100.00 & 0.00 & 1.000000 & 100.00 & 0.00 \\
\hline $2 \mathrm{C}$ & ${ }^{(\mathrm{H})} 1,2,3,4,4,4,4,4,4,5,6,7^{(\mathrm{T})}$ & 0.955590 & 82.99 & 17.01 & 1.048739 & 82.99 & 17.01 \\
\hline $2 \mathrm{D}$ & (H) $3,4,5,8,8,8,8,8,8,8,8,8^{(\mathrm{T})}$ & 0.975000 & 90.24 & 9.76 & 1.026316 & 90.24 & 9.76 \\
\hline $2 \mathrm{E}$ & ${ }^{(\mathrm{H})} 8,8,8,8,8,8,8,8,8,11,12,13^{(\mathrm{T})}$ & 0.980447 & 92.33 & 7.67 & 1.020349 & 92.33 & 7.67 \\
\hline $2 \mathrm{~F}$ & (H) $2,7,7,7,7,7,7,7,7,7,7,35^{(\mathrm{T})}$ & 0.938754 & 76.92 & 23.08 & 1.069793 & 76.92 & 23.08 \\
\hline $2 \mathrm{G}$ & (H) $7,7,7,7,7,7,7,7,7,7,7,35^{(\mathrm{T})}$ & 0.949772 & 80.87 & 19.13 & 1.055839 & 80.87 & 19.13 \\
\hline $2 \mathrm{H}$ & (H) $2,2,2,2,2,2,9,9,9,9,9,9^{(\mathrm{T})}$ & 0.931596 & 74.39 & 25.61 & 1.079245 & 74.39 & 25.61 \\
\hline $2 \mathrm{I}$ & ${ }^{(\mathrm{H})} 2,2,2,2,2,2,7,7,7,7,7,7^{(\mathrm{T})}$ & 0.939759 & 77.27 & 22.73 & 1.068493 & 77.27 & 22.73 \\
\hline $2 \mathrm{~J}$ & (H) $4,4,4,4,4,4,4,4,4,4^{(\mathrm{T})}$ & 1.000000 & 100.00 & 0.00 & 1.000000 & 100.00 & 0.00 \\
\hline $2 \mathrm{~K}$ & (H) $4,4,4,4,4,8,8,8,8,8^{(\mathrm{T})}$ & 0.963504 & 85.92 & 14.08 & 1.039370 & 85.92 & 14.08 \\
\hline $2 \mathrm{~L}$ & (H) $4,4,4,4,4,16,16,16,16,16^{(\mathrm{T})}$ & 0.936170 & 76.00 & 24.00 & 1.073171 & 76.00 & 24.00 \\
\hline $2 \mathrm{M}$ & (H) $4,4,4,4,4,64,64,64,64,64^{(\mathrm{T})}$ & 0.908870 & 66.59 & 33.41 & 1.111441 & 66.59 & 33.41 \\
\hline $2 \mathrm{~N}$ & (H) $4,4,4,4,4,152,152,152,152,152^{(\mathrm{T})}$ & 0.902683 & 64.53 & 35.47 & 1.120836 & 64.53 & 35.47 \\
\hline 20 & (H) $4,4,4,4,4,256,256,256,256,256^{(\mathrm{T})}$ & 0.900787 & 63.90 & 36.10 & 1.123772 & 63.90 & 36.10 \\
\hline $2 \mathrm{P}$ & (H) $4,4,4,4,4,3.999,3.999,3.999,3.999,3.999^{(\mathrm{T})}$ & 1.000014 & 99.99 & 0.01 & 0.999986 & 99.99 & 0.01 \\
\hline $2 \mathrm{Q}$ & ${ }^{(\mathrm{H})} 4,4,4,4,4,4.001,4.001,4.001,4.001,4.001^{(\mathrm{T})}$ & 0.999986 & 99.99 & 0.01 & 1.000014 & 99.99 & 0.01 \\
\hline $2 \mathrm{R}$ & (H) $4,4,4,4,4,-4,-4,-4,-4,-4^{(\mathrm{T})}$ & 0.000000 & -100.00 & 100.00 & 0.000000 & -100.00 & 100.00 \\
\hline $2 \mathrm{~S}$ & (H) $-8,-8,-8,-8,-8,4,4,4,4,4^{(\mathrm{T})}$ & 1.517241 & 18.92 & 81.08 & 0.745763 & 18.92 & 81.08 \\
\hline $2 \mathrm{~T}$ & (H) $-16,-16,-16,-16,-16,4,4,4,4,4^{(\mathrm{T})}$ & 1.233645 & 45.05 & 54.95 & 0.840764 & 45.05 & 54.95 \\
\hline $2 \mathrm{U}$ & (H) $-64,-64,-64,-64,-64,4,4,4,4,4^{(\mathrm{T})}$ & 1.147826 & 59.04 & 40.96 & 0.885906 & 59.04 & 40.96 \\
\hline $2 \mathrm{~V}$ & (H) $-152,-152,-152,-152,-152,4,4,4,4,4^{(\mathrm{T})}$ & 1.175519 & 54.01 & 45.99 & 0.897905 & 62.95 & 37.05 \\
\hline $2 \mathrm{~W}$ & (H) $-256,-256,-256,-256,-256,4,4,4,4,4^{(\mathrm{T})}$ & 1.184599 & 52.48 & 47.52 & 0.901785 & 64.23 & 35.77 \\
\hline $2 \mathrm{X}$ & (H) $-3.999,-3.999,-3.999,-3.999,-3.999,4,4,4,4,4^{(\mathrm{T})}$ & 0.925214 & 72.17 & 27.83 & 0.779517 & 27.74 & 72.26 \\
\hline $2 \mathrm{Y}$ & (H) $-4.001,-4.001,-4.001,-4.001,-4.001,4,4,4,4,4^{(\mathrm{T})}$ & 0.925254 & 72.18 & 27.82 & 0.779538 & 27.74 & 72.26 \\
\hline
\end{tabular}


Optinalytic (Statistical) Mirroring

\section{Appendix B}

Table B1: Results of Statistical Mirroring

\begin{tabular}{llll}
\hline & Kc & \%Sim. & \% Dsim. \\
\hline Population A (Meanic) & 0.926674 & 72.67 & 27.33 \\
Population B (Meanic) & 0.892851 & 61.29 & 38.71 \\
Population C (Meanic) & 0.917956 & 69.67 & 30.33 \\
Population D (Meanic) & 0.944487 & 78.96 & 21.04 \\
Population A (Medianic) & 0.961813 & 85.29 & 14.71 \\
Population B (Medianic) & 1.003115 & 98.77 & 1.23 \\
Population C (Medianic) & 0.91438 & 68.45 & 31.55 \\
Population D (Medianic) & 0.97002 & 88.36 & 11.64 \\
Population A (Modalic) & 0.809812 & 36.08 & 63.92 \\
Population B (Modalic) & 0.774372 & 26.36 & 73.64 \\
Population C (Modalic) & 0.817832 & 38.36 & 61.64 \\
Population D (Modalic) & 0.840866 & 45.09 & 54.91 \\
Population A (Maximalic) & 0.809812 & 36.08 & 63.92 \\
Population B (Maximalic) & 0.774372 & 26.36 & 73.64 \\
Population C (Maximalic) & 0.817832 & 38.36 & 61.64 \\
Population D (Maximalic) & 0.840866 & 45.09 & 54.91 \\
Population A (Minimalic) & 1.18849 & 51.84 & 48.16 \\
Population B (Minimalic) & 1.316043 & 35.11 & 64.89 \\
Population C (Minimalic) & 1.431115 & 24.81 & 75.19 \\
Population D (Minimalic) & 1.259717 & 41.61 & 58.39 \\
\hline
\end{tabular}


Optinalytic (Statistical) Mirroring

\section{Appendix C}

Table C1: Results of Statistical Mirroring

\begin{tabular}{rrrrrrr}
\hline & \multicolumn{3}{c}{$\boldsymbol{x}(\boldsymbol{A}):(\boldsymbol{A})$} & & $\boldsymbol{x}(\boldsymbol{A}):(\boldsymbol{A})$ & \\
\hline & $\mathbf{K c}$ & \% Dsim. & \%Sim. & Kc & \% Dsim. & \% Sim. \\
$\times \mathbf{2}$ & 1.200000 & 50.00 & 50.00 & 1.200000 & 50.00 & 50.00 \\
$\times \mathbf{3}$ & 1.333333 & 66.67 & 33.33 & 1.333333 & 66.67 & 33.33 \\
$\times \mathbf{4}$ & 1.428571 & 75.00 & 25.00 & 1.428571 & 75.00 & 25.00 \\
$\times \mathbf{5}$ & 1.500000 & 80.00 & 20.00 & 1.500000 & 80.00 & 20.00 \\
$\times \mathbf{6}$ & 1.555556 & 83.33 & 16.67 & 1.555556 & 83.33 & 16.67 \\
$\times \mathbf{7}$ & 1.600000 & 85.71 & 14.29 & 1.600000 & 85.71 & 14.29 \\
$\times \mathbf{8}$ & 1.636364 & 87.50 & 12.50 & 1.636364 & 87.50 & 12.50 \\
$\times \mathbf{9}$ & 1.666667 & 88.89 & 11.11 & 1.666667 & 88.89 & 11.11 \\
$\times \mathbf{1 0}$ & 1.692308 & 90.00 & 10.00 & 1.692308 & 90.00 & 10.00 \\
& & $\boldsymbol{x}(\boldsymbol{A}):(\boldsymbol{B})$ & & & $\boldsymbol{x}(\boldsymbol{A}):(\boldsymbol{B})$ & \\
$\times \mathbf{1} / \mathbf{2}$ & 0.857143 & 50.00 & 50.00 & 0.857143 & 50.00 & 50.00 \\
$\times \mathbf{1} / \mathbf{3}$ & 0.800000 & 66.67 & 33.33 & 0.800000 & 66.67 & 33.33 \\
$\times \mathbf{1} / \mathbf{4}$ & 0.769231 & 75.00 & 25.00 & 0.769231 & 75.00 & 25.00 \\
$\times \mathbf{1} / \mathbf{5}$ & 0.750000 & 80.00 & 20.00 & 0.750000 & 80.00 & 20.00 \\
$\times \mathbf{1} / \mathbf{6}$ & 0.736842 & 83.33 & 16.67 & 0.736842 & 83.33 & 16.67 \\
$\times \mathbf{1} / \mathbf{7}$ & 0.727273 & 85.71 & 14.29 & 0.727273 & 85.71 & 14.29 \\
$\times \mathbf{1} / \mathbf{8}$ & 0.720000 & 87.50 & 12.50 & 0.720000 & 87.50 & 12.50 \\
$\times \mathbf{1} / \mathbf{9}$ & 0.714286 & 88.89 & 11.11 & 0.714286 & 88.89 & 11.11 \\
$\times \mathbf{1} / \mathbf{1 0}$ & 0.709677 & 90.00 & 10.00 & 0.709677 & 90.00 & 10.00 \\
\hline
\end{tabular}

